\title{
A needs assessment for a graduate level course in optical networking
}

\section{Qi Zhang, Jana Reisslein, James Klein, Martin Reisslein}

Qi Zhang, Jana Reisslein, James Klein, Martin Reisslein, "A needs assessment for a graduate level course in optical networking," Proc. SPIE 9663, Eighth International Topical Meeting on Education and Training in Optics and Photonics, 966310 (6 October 2003); doi: 10.1117/12.2207482

SPIE Event: Eighth International Topical Meeting on Education and Training in Optics and Photonics, 2003, Tucson, Arizona, United States 


\title{
A needs assessment for a graduate level course in optical networking
}

\author{
Qi Zhang, Jana Reisslein, James Klein \\ Educational Technology, Division of Psychology in Education, College of Education, Arizona State University, Tempe, AZ 85287-0611 \\ qiggzhang@asu.edu, Jana.Reisslein@asu.edu, James.Klein@asu.edu \\ Martin Reisslein \\ Dept. of Electrical Engineering, Ira A. Fulton School of Engineering, Arizona State University, Tempe, AZ 85287-5706 \\ reisslein@asu.edu
}

\begin{abstract}
To explore the need for a graduate level engineering course in optical networking, a needs assessment consisting of (i) an investigation of 14 existing optical networking courses, (ii) an analysis of online surveys among the networking community and the ASU electrical engineering department, (iii) faculty interviews, and (iv) focus groups was conducted. Survey responses from a total of 61 respondents were received and analyzed. The results support the need for a graduate level course in optical networking. Our analyses indicate that a graduate course in optical networking should (i) focus on the basic mechanisms and current trends in optical networking, (ii) be based on a text book and instructor slides combined with collections of examples and problems. Regarding the optimal delivery method it was found that current students and faculty strongly prefer face-to-face delivery complemented by on-line readings and assignments, whereas working engineering professionals are more open to the idea of online courses.

(c)2003 Optical Society of America

OCIS codes: (000.2060) Education; (060.4250) Networks
\end{abstract}

\section{Definition and Purpose}

New bandwidth intensive telecommunications services, such as multimedia streaming entertainment, distance education, and telemedicine, require increased capacities in the Internet. It is widely believed in the networking engineering community that only fiber optics will be able to provide the enormous bandwidth required for future telecommunications services. For this reason, optical networking has been intensively explored in the networking research community over the past two decades and continues to be the focus of intense research efforts [1]. The outcomes of these research efforts are presented at the major networking conferences, such as IEEE Infocom, IEEE Globecom, and the IEEE International Conference on Communications in specialized optical networking sessions and workshops, which are very popular. In fact, for the past three years, the optical networking workshop being held during the IEEE International Conference on Communications has attracted the largest registration number among the ICC workshops [2]. In addition, specialized optical communications and networking conferences have enjoyed large attendance over the past several years, with the largest conference, the Optical Fiber Conference attracting over 10,000 researchers and engineers every year. Furthermore, recent years have seen the emergence of specialized optical networking journals and magazines, such as the Optical Networking Magazine, which was launched by Kluwer Academic Publishers in 1999, and the increasing number of networking articles in the IEEE/OSA Journal of Lightwave Technology. These extensive research and publication efforts indicate that optical networking is establishing itself as an important sub-discipline of networking.

Reflecting this trend, many electrical engineering graduate programs in the U.S have begun to offer courses on optical networking. However, in numerous institutions optical networking courses are not included in the core graduate engineering curriculum. As part of the effort to develop and expand the electrical engineering (EE) program at Arizona State University (ASU), it is needed to gain an insight into the need of a graduate course in optical networking at ASU, and what the optimal instructional contents, and optimal delivery method of the course would be.

To address this question, a needs assessment was conducted. A secondary goal was to investigate the attitudes of the broad engineering community (students, faculty, and engineering professionals) toward a graduate level optical networking course in general. The needs assessment was designed to answer the following questions: 1. Is it necessary to offer a graduate course in optical networking in the electrical engineering department at ASU? 2. What are the general attitudes of students, faculty, and engineering professionals toward a graduate optical networking 
course? 3. What would be the optimal instructional content, teaching materials, and delivery method for a graduate optical networking course?

\section{Method}

The methods and techniques in this needs assessment followed Rossett's [3] suggestions for conducting a needs assessment. A comprehensive approach involving a combination of methods was employed. The following section provides the description of the various data sources, numerous data collection instruments, and the needs assessment participants.

\section{Data sources and Instruments}

\subsection{Extant data}

Extant data were obtained from optical networking and fiber optics graduate courses across the country. To get a nationwide picture of the optical networking field, syllabi and course descriptions for optical networking and fiber optics courses offered at other universities, mostly top EE graduate schools or equivalents to ASU were reviewed to identify the course goals, learning objectives, and contents. The syllabi and course descriptions were obtained from Columbia University, Northwestern University, University of Texas at Arlington, San Jose State University, University of Maryland, Virginia Tech, Massachusetts Institute of Technology, University of Hawaii, University of California at Davis, University of Pennsylvania, University of Arizona, Carnegie Mellon, Purdue University, and Iowa State University.

Based on the analysis of the obtained graduate optical networking and fiber optics curricula, syllabi, and course descriptions, a set of common learning goals and major content areas were identified. In addition, specific topics and research areas were extracted from the extant data. From this extant data analysis we created a list of the major optical networking and fiber optics topics that are being taught at the graduate engineering level. We compiled a set of questions to be used in an online survey to be distributed to the general engineering community. The initial set of questions (topic areas) was further clarified and refined as a result of interviews with experts from the optical networking and fiber optics field.

\subsection{Online survey}

The online survey was designed to determine the general perception of the importance of optical networking in the graduate engineering curriculum and the optimal content, delivery method, and teaching materials for a graduate optical networking course. The survey was distributed through the IEEE Technical Committee on Computer Communications (TCCC) mailing list, which reaches close to one thousand networking engineering professionals, students, and researchers across the U.S, and the mailing list of the electrical engineering department at ASU. The survey employed a four-point Likert type rating scale with the strongly agree category equaling a numerical value of 3 and strongly disagree equaling a numerical value of 0 in ranking the agreement with the individual statements. The survey required the respondents to rank (strongly agree, agree, disagree, strongly disagree) thirty-nine statements that covered the issues of the optimal (1) prerequisite knowledge that students should have before taking a graduate level optical networking course, (2) general focus areas of an optical networking course, (3) topics in Architectures and Hardware, (4) topics in Protocols, (5) topics in General Principles, (6) teaching materials and strategies, (7) course delivery method, and (8) the necessity of optical networking in graduate engineering curriculum in general. The respondents also indicated their current position as either a graduate student, faculty or engineering professional. The respondents were given the opportunity to provide additional comments and elaborations of their answers (rankings). These elaborations were considered as qualitative data source. The outline of the survey is enclosed in the Appendix.

\subsection{Interview}

Interviews were conducted with two faculty members (the chair of the graduate program and an assistant professor who is conducting research in optical networking) in the EE department at ASU. The goal was to explore the attitudes of a sample of the members of the EE department toward an optical networking graduate course.

Specifically, questions targeted the perceived need for and students' interest in a graduate optical networking course, the available support (physical, personnel, and educational resources) for implementing a graduate optical 
networking course, and the perceived importance of a graduate course in optical networking for the general goals of the EE department at ASU. The interview encompassed role-specific questions such as "How would the optical networking course fit in the current EE graduate curriculum?" for the chair of the graduate program, and "What would be the focus of a graduate course in optical networking if you were to teach it?" for the assistant professor. Each of the interviewees responded to a set of ten questions. The approximate duration of each of the interviews was 45 minutes.

\subsection{Focus group}

A focus group was conducted with a sample of graduate students in the EE department at ASU. The purpose of the focus group was to determine students' attitudes toward the potential course in optical networking, their perspectives on the optimal contents, instructional methods, and methods of delivery. The focus group participants responded to eight questions covering their knowledge of and interest in optical networking, the preferred course content, and delivery method. The questions were role-specific, such as "Are you taking or planning to take a graduate optical networking course? Explain why?". The focus group discussion lasted approximately 1.5 hours and the obtained qualitative data provided more in-depth insights into the role of optical networking in the graduate engineering curriculum.

\section{Participants}

A total of sixty-one responses were received from the online survey. The entire nationwide sample consisted of twenty faculty members, thirty-five graduate students, and six engineering professionals. The survey was distributed over the IEEE TCCC mailing list and over the ASU EE mailing list. The aim was to reach the broad networking engineering community (faculty, graduate students, engineering professionals) as well as the specific ASU EE community. The survey did not require biographical information except for the respondent's current occupation. Approximately one half of the online survey responses were obtained from the ASU respondents. This constitutes approximately $52 \%$ of the gross sixty-one responses nationwide.

A total of thirty-two respondents from the EE department at ASU participated in the online survey that was distributed through the ASU EE mailing list. Specifically, six EE faculty members (approximately $18 \%$ of all ASU survey response rate) responded to the online survey. Twenty-five ASU EE graduate students responded to the online survey, counting approximately $78 \%$ of the overall ASU survey response rate. In addition, one ASU respondent was an engineering professional.

Two faculty members of the EE department at ASU were interviewed. One of the faculty members was the chair of the graduate program in the EE department. The other faculty member was a junior faculty who actively conducts research in optical networking and is considering teaching a course in optical networking.

Five graduate students from the EE department at ASU were interviewed during a focus group session. Two of the graduate students have strong research interest in the optical networking area and conduct research in the optical networking field. The students ranged in their experience in optical networking from beginner (first year Ph.D.) to advanced (Ph.D. candidate). All the students were enrolled in the Ph.D. engineering program.

\section{Quantitative Results}

In this section, the most important quantitative results of the needs assessment are presented, specifically the results from the online survey distributed nationwide are presented. The results are organized by the main areas that this needs assessment addresses. Generally, the survey data obtained from the ASU respondents are consistent with those of the respondents from the broader engineering community. Minor differences are pointed out in the discussion. Note that the rating scale implemented in the online survey ranged in numerical values from 3 (strongly agree) to 0 (strongly disagree).

\subsection{General need}

Nationwide, 51 out of the total of 61 respondents (84\%) agreed or strongly agreed with the addition of an optical networking course to the engineering graduate curriculum $(M=2.47)$. Specifically, 30 respondents strongly agreed, 21 agreed, and four disagreed. There was no strong disagreement.

Table 1. depicts the differences in perceptions of the importance of an optical networking course in the graduate engineering curriculum. The data suggest that engineering professionals have the strongest preference. 
Table 1. Overall need for an optical networking graduate engineering course

\begin{tabular}{ll}
\hline Current Status & Mean \\
\hline Students & 2.51 \\
Faculty & 2.31 \\
Professionals & 2.75 \\
\hline
\end{tabular}

The extracted results of the online survey of ASU EE graduate students, faculty, and an engineering professional support the idea of offering a graduate level course in optical networking at ASU $(M=2.35)$. Specifically, approximately $90 \%$ of the all the respondents $(\underline{\mathrm{n}}=32)$ either agreed $(\underline{\mathrm{n}}=14)$ or strongly agreed $(\underline{\mathrm{n}}=$ 14) that there is a need for an optical networking graduate course at ASU.

\subsection{Course focus}

As shown in Figure 1., the responses from the ASU survey and the nationwide survey (aggregates reported) indicate that a course focus on "Understanding of basic mechanism in optical networking" was ranked the highest (aggregate $M=2.59$ ), followed by "Understanding of current trends in optical networking" as the second highest (aggregate $M=2.45$ ). The lowest ranked course focus area was "Designing and evaluating protocols for optical networks" (aggregate $M=2.21$ ). All the course focus areas had a mean score larger than 2.0 ( $M$ ranging from 2.21 to 2.59), indicating an agreement with having these areas to be the course focus.

It is interesting to note that all the respondents (faculty, students, engineering professionals) ranked the focus areas approximately equally. However, the engineering professionals had slightly higher preference for more practically oriented focus areas compared to faculty. Specifically, the mean score for faculty for the focus area "Designing optical networking architectures" was 2.05 compared to the mean score of 2.83 for the engineering professionals. The same trend ( $M=2.15$ for faculty and $M=2.83$ for the engineering professionals) was observed in the focus area "Designing and evaluating protocols for optical networks".

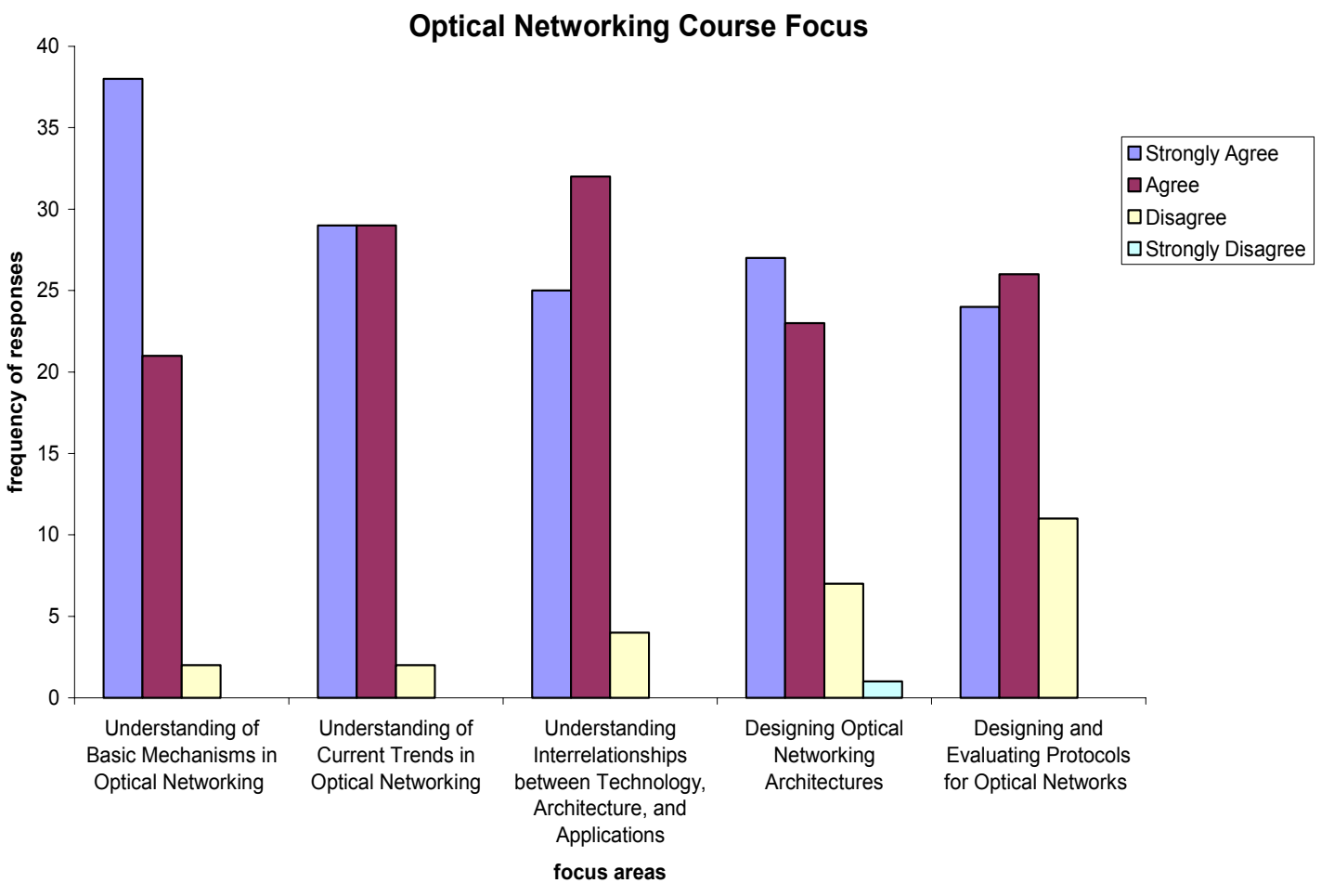

Fig. 1. Optical networking course focus 


\subsection{Optimal content}

The syllabi and course descriptions from the 14 universities across the US were analyzed. It was found that the main course content includes architectures and hardware, access, scheduling methods, protocols, control and management, protection, and WDM. The content topics and frequency are summarized in Table 2.

Table 2. Suggested topics and frequency

\begin{tabular}{ll}
\hline Topic & Frequency \\
\hline Architectures and Hardware & 12 \\
Access & 10 \\
Scheduling Methods & 10 \\
Protocols & 8 \\
Control and Management & 6 \\
Protection & 6 \\
WDM & 5 \\
\hline
\end{tabular}

Next, we present the analysis of the survey responses on the course topics. The topics falling under the General Principles were ranked as the most important content to cover in an optical networking course (mean score ranged from 2.40 to 2.81, aggregate overall $M=2.55$ ). The ranking of the subtopics within the General Principles topic was in the order from highest to lowest importance given by: WDM/ DWDM; layered/hierarchical network architecture; $1^{\text {st }}$ to $3^{\text {rd }}$ generation of optical systems. This ranking appears to indicate the principles that are valid for all optical networks (WDM/DWDM and layered/hierarchical network architecture) are considered more important than the historical overview of the development from $1^{\text {st }}$ to $3^{\text {rd }}$ generation optical systems.

The other content areas and topics within optical networking were also ranked as important, specifically, the aggregate mean scores for the topics within the Architectures and Hardware area ranged from 2.28 to 2.49 (overall $M=2.39$ ), for the Protocols topics the aggregate mean ranged from 2.16 to 2.68 (overall $M=2.44$ ).

The respondents of the survey mailed on the ASU EE mailing list rated the three main content areas (Architectures and Hardware, Protocols, and General Principles) roughly as equally important. The overall combined mean scores for these three content areas on the survey was 2.41 (Architectures and Hardware 2.42, Protocols 2.39, and General Principles 2.53). This indicates a rating that falls between the agree (2) and strongly agree (3) category.

\subsection{Delivery method}

In terms of the optimal delivery method, the survey respondents ranked an Entirely Face-to-Face (traditional) method of course delivery as the most optimal $(M=2.38)$. The respondents also highly ranked the hybrid (Emphasize Face-to-Face Classroom Activities with Online Readings and Assignments) form of course delivery ( $M$ $=2.33$ ). The aggregate online survey results show a large gap between the mean scores of the most favorite delivery method (Exclusively Face-to-Face, $M=2.38$ ) and least favorite delivery method (Entirely-Online, $M=0.58$ ). $88 \%$ of the respondents explicitly disagreed (27\%) or strongly disagreed (61\%) with delivering the course entirely online.

The data obtained solely from the ASU respondents are consistent with the general results of the nationwide online survey. The ASU survey showed a large margin between the most and the least favorite delivery method. $90 \%$ of the ASU respondents preferred the course to be delivered exclusively face-to-face, and $80 \%$ of the ASU respondents explicitly disagreed (27\%) or strongly disagreed (53\%) with the entirely online course delivery method. The mean score rating for the Exclusively Face-to-Face course delivery method was 2.50, which sharply contrasts with the mean rating of 0.77 for the Entirely Online course delivery method.

It is interesting to note that the hybrid course delivery method, which emphasizes face-to-face classroom activities but combines those with online readings and assignments, received a relatively high ranking (national aggregate $M=2.33$, ASU $M=2.17$ ). This delivery method is aligned with the ASU distance education goals (to become a leader in distance education). The assumption is that this hybrid course delivery method would improve accessibility, resource utilization, and revenue. Second, from the limited overall sample of the engineering professionals $(\underline{\mathrm{n}}=6)$, it appears that the engineering professionals have higher preference for entirely online instruction $(M=1.00)$ than the graduate engineering students $(M=0.47)$. The differences among the three groups (faculty, students, and engineering professionals) can be observed in Figure 2. 


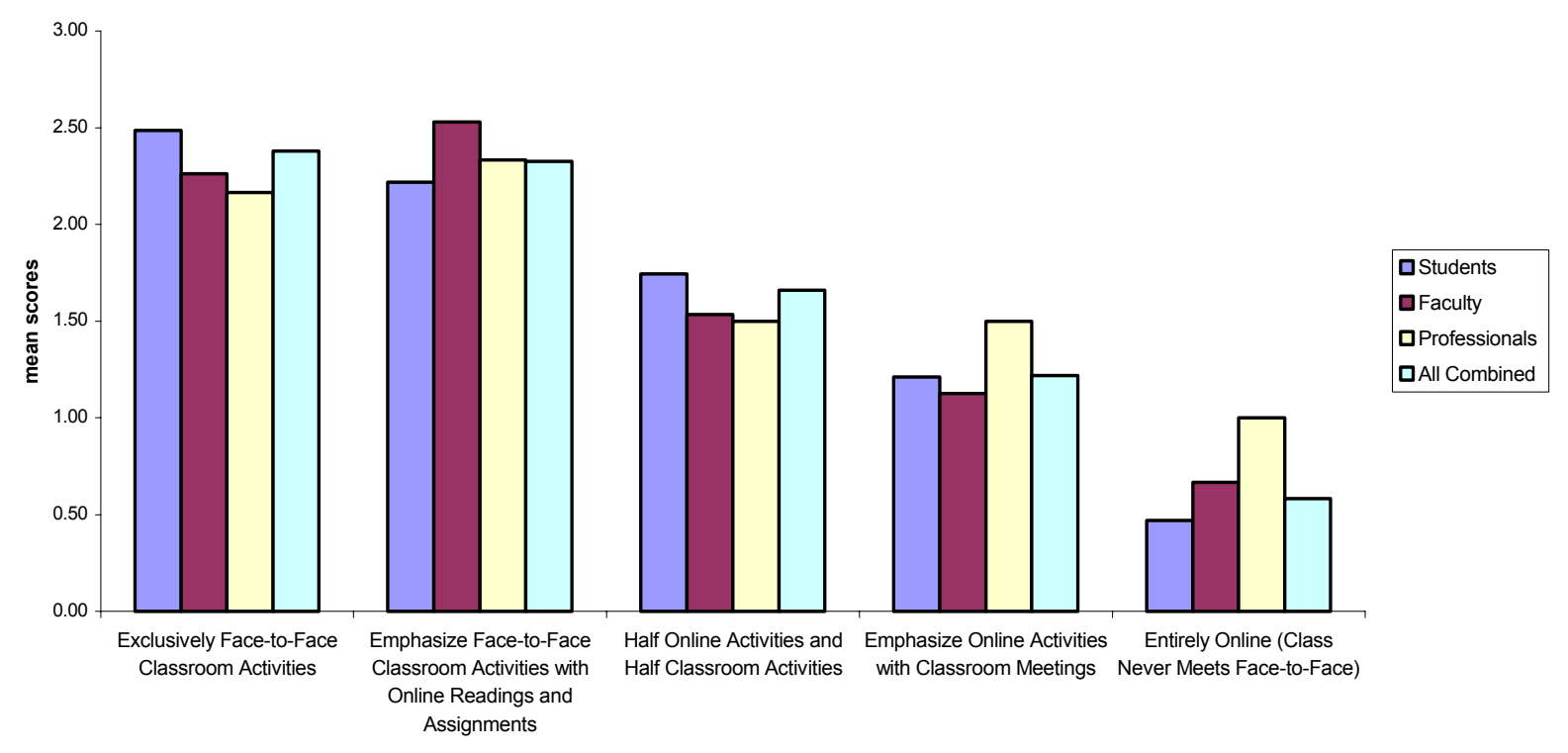

\subsection{Teaching materials}

Fig. 2. Optimal delivery method for an optical networking course

The preferences for teaching materials expressed in the online surveys are illustrated in Figure 3. The textbook received the highest rating from the online survey participants $(M=2.63)$. There is a slight difference between ASU and the rest of the national sample in the second preferred teaching material. The ASU respondents preferred the "Collection of Examples (real models, animated applets)" $(M=2.57)$ whereas in the nationwide survey, "Instructor Slides (contents)" were ranked as the second highest $(M=2.53)$. The Database of Test Questions was generally ranked the lowest $(M=1.86)$.

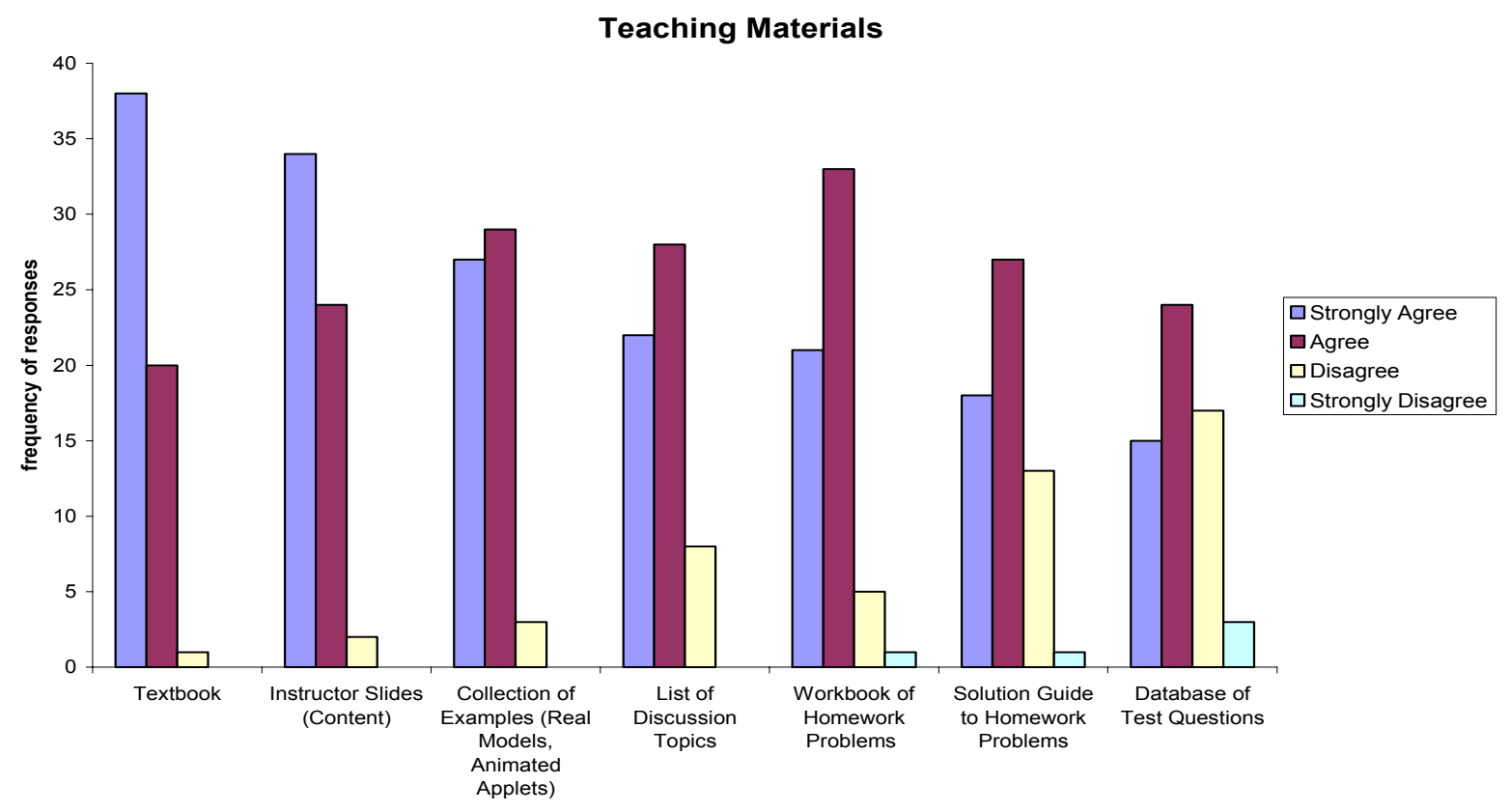

Fig. 3. Optical networking teaching materials / strategies 


\subsection{Prerequisite knowledge}

In terms of the prerequisite knowledge that students need to acquire before taking a graduate course in optical networking, the online survey respondents rated Networking Basics (TCP/IP) as the most important $(M=2.41)$. The second highest ranked prerequisite knowledge was Fiber Optics Basics (Lasers, Detectors, Fibers) with $M=$ 1.95. This was followed by Performance Evaluation Basics (Stochastics, Simulation) prerequisite knowledge $(M=$ 1.90). The second to last in prerequisite knowledge importance was ranked Wave Physics $(M=1.38)$. The lowest rating of prerequisite knowledge was Advanced Networking (MPLS, Differentiated Services) with $M=1.31$.

An interesting finding emerged during the analysis of the group (faculty, students, engineering professionals) differences. Faculty rated the fiber optics basics (lasers, detectors, fibers) topic as not very important prerequisite knowledge $(M=1.55)$. This result contrasts with ratings of the same prerequisite knowledge area by the engineering professionals $(M=2.50)$.

\section{Qualitative Results}

The presentation of the qualitative data includes the analysis of the extant data, the analysis of the qualitative responses and the comments obtained from the online survey, the comments collected during interviews with the subject matter experts, and the observations from the focus group with graduate engineering students.

\subsection{Extant data}

The analysis of the extant data (optical networking course syllabi and course descriptions from 14 universities across the US) revealed that the main content in a graduate level course in optical networking covers a broad spectrum, including architectures and hardware, access, scheduling methods, protocols, control and management, protection, and wavelength division multiplexing (WDM). The majority of the courses included homeworks, lab sessions/demonstrations, and projects (applied or theoretical). The homeworks were intended to reinforce the concepts learned in classes. All the courses had exams as an assessment method of student performance. The most commonly used textbooks were Optical Networks: A Practical Perspective [4], Fiber Optic Communication Systems [5], and Optical Communication Networks [6].

\subsection{Qualitative data analysis}

The qualitative data from the write-in comment fields in the online survey suggest that the respondents strongly support the idea of offering a graduate level course in optical networking for engineering students. Respondents generally consider optical networking as an emerging technology that meets the demand of high-speed communication and cost reduction. Optical networking is perceived as the future of networking, replacing copper wire networks. One respondent sees the optical communication industry as the "latest job-providing field well worthy of exploration." The understanding of optical networking is viewed essential to engineers in the industry. Accordingly, a graduate level course in optical networking would help prepare the students for the future; therefore good engineering programs should offer such a course.

In terms of the instructional content, the respondents would like the students to be aware of the state-of-the-art technology on the market. Because optical networking has a broad range of topics, it is important that the instructors clearly state the course focus. A few respondents would prefer to include an undergraduate preparatory course or to cover topics such as routing, flow control, and networking in a general scope engineering course.

Flexibility should be given to students if they are interested in a particular topic, including making this course an elective instead of a core course. It was suggested that conference and journal papers should be included as teaching materials.

\subsection{Interview}

The interviews with two ASU EE professors indicate that optical networking is an emerging area of research interest. It appears that the EE department at ASU is generally optimistic about the idea of offering a graduate course in optical networking. Numerous faculty members conduct research in optical networking and related fields. The interviewees felt that the topic of optical networking fits well into the graduate engineering curriculum at ASU and might be a worthwhile addition to the engineering program. The interviewees perceived the EE department as 
having sufficient resources (facilities, personnel, equipment, instructional support, etc.) that would be available for a graduate course in optical networking.

The interviewees agreed that students should have some basic understanding of general networking principles and light physics before taking a graduate course in optical networking. There was a consensus between the interviewees in regard to the course focus. The course should focus on the design, analysis, and implementation of optical networks. Furthermore, the course should cover optical networks architectures and hardware, protocols, and specialized topics.

There were some concerns expressed by the interviewees in regard to the potential enrollment in the course. Specifically the number of students who would be interested in the course might be low due to the currently limited employment opportunities in the optical networking field, which is currently in a recession. However, both interviewees are positive about the future of the optical communications and networking industry and foresee the market as improving, thus leading to increased interest in optical networking education among graduate students as well as job opportunities in the optical networking area.

\subsection{Focus group}

The focus group with five ASU EE graduate students indicates that optical networking is still an emerging research area. Generally, the students did not know extensively about optical networking. They had some basic knowledge that they gained in undergraduate engineering classes. Two of the respondents had a high degree of specialized knowledge in optical networking, since they selected the field as an area of active research and study. The students expressed interest in introductory as well as advanced / specialized contents in optical networking. However, those students who did not select optical networking as a research area would be only partially interested in taking a formal graduate course in optical networking. This is due to the course content not being compatible with personal research interests, and the currently limited market demand for professionals in the optical communications and networking field. All students reported their preference for the lecture delivery format, which they perceived as an efficient way of content delivery. The students also valued projects and problem-solving scenarios, since they might be a better measure of performance. In addition, the students perceived that they learn more by actually producing something than by recalling information on a quiz. The students preferred the face-to-face course delivery method. In their opinion, this instruction allows for enhanced interaction among the learners, and promotes the studentprofessor interaction. This is consistent with the findings from the online survey. In addition, the students were able to recognize some of the advantages of distance education, especially flexibility. However, they were concerned about the available technical support for a distance education course, and interactivity. (This concern may in part stem from the quite extensive technical difficulties and outages that student IT system at ASU had experienced in the fall 2002 and spring 2003 semesters.) The students felt strongly about the option to have face-to-face meetings with the instructor even if the course was offered in the distance education (online) format.

\section{Implications and Recommendations}

Overall, the collected data indicate that there is a need for the ASU EE department to offer a graduate course in optical networking. Specifically, the data from the ASU respondents suggest that $90 \%$ of the respondents either agreed or strongly agreed that there is a need for an optical networking graduate course at ASU. None of the respondents reported strong disagreement. Such need is echoed nationwide. Overall, the majority $(84 \%)$ of the sample of the engineering community perceived optical networking as a necessary part of graduate engineering curriculum. The ASU EE department has sufficient resources, including facilities, personnel, and instructional support to support the implementation of a graduate course in optical networking.

The collected qualitative data indicate that optical networking should be included in graduate engineering programs. The attitudes toward optical networking in general were very positive among all of the respondents. The sample from the engineering community (students, faculty, and engineering professionals) reported that optical networking should be taught in graduate engineering schools to better prepare students for the future. The engineering community perceives optical networking as an emerging area of research that is likely to grow; given the communication industry comes out of the currently experienced recession.

In terms of the contents of a graduate level course in optical networking, it is recommended that the following should be covered: (i) General Principles (in the descending order of importance: WDM/DWDM; layered/hierarchical network architecture; 1 st to 3rd generation of optical systems), (ii) Protocols (in the descending order of importance: routing and wavelength assignment; protection and restoration; switching; TCP/IP over WDM; medium access; grooming; MPLS), and (iii) Architectures and Hardware (in the descending order of importance: 
switches/amplifiers/routers; mesh; ring; single-hop; access/metro network; backbone). The results support the idea of broad instructional content being taught in a graduate level course in optical networking.

Since a textbook is used / considered as the primary tool for supporting teaching, it is suggested that a graduate optical networking course is based on a textbook. The data also indicate that the course instructors could include slides (to present content) to support teaching. Therefore, it might be advisable to design and develop presentation slides to impart knowledge and to reinforce the most important content. Those presentation slides would be aligned with the textbook and the overall course objectives to facilitate learning.

Based on the analyzed data, it is recommended that a graduate course in optical networking is delivered either by traditional means (exclusively face-to-face instruction) or in a hybrid form, which emphasizes face-to-face classroom activities but combines those with online readings and assignments. However, considering the high rate of disagreement, it is not recommended to use an entirely online format of a course delivery.

\section{References}

Report of NSF Workshop on Fundamental Research in Networking, 2003. http://www.cs.virginia.edu/ jorg/workshop1/ El-Bawab, T, "Optical networking in the Metro Area", Optical Networks Magazine, 115-116 (2001).

Rossett, A., Training Needs Assessment (Educational Technology Publications, Englewood Cliffs, NJ 1987).

Ramaswami, R., Sivarajan, K.N., Optical Networks: A Practical Perspective, $2^{\text {nd }}$ edition (Morgan Kaufmann, 2002).

Agrawal, G. P., Fiber Optic Communication Systems, $3^{\text {rd }}$ edition (John Wiley \& Sons, 2002).

Mukherjee, B., Optical Communication Networks (McGraw Hill, 1997).

\section{Appendix}

Online survey form 
The purpose of this survey is to collect data on the role of Optical networking in graduate instruction. Please fill out the survey by checking the options that best represent your opinions for each statement. Your input is very valuable.

$$
\text { SA - Strongly Agree A - Agree D - Disagree SD - Strongly Disagree }
$$

1. The prerequisite knowledge a student should have before taking a course in Optical networking is:

Networking Basics (TCP/IP)

Fiber Optics Basics (Lasers, Detectors, Fibers)

SA A D SD

Performance Evaluation Basics (Stochastics, Simulation)

SA A D SD

Wave Physics

Advanced Networking (MPLS, DiffServ)

SA A D SD

SA A D SD

SA A D SD

2. The focus of a course in Optical networking should be on knowledge/skills in:

Understanding of Basic Mechanisms in Optical networking

SA A D SD

Understanding of Current Trends in Optical networking

Understanding Interrelationships between Technology, Architecture, and Applications

Designing Optical networking Architectures

Designing and Evaluating Protocols for Optical Networks

SA A D SD

SA A D SD

SA A D SD

SA A D SD

3. Which of the following ARCHITECTURES \& HARDWARE topics should students learn?

Single-Hop (Star)

Ring

Mesh

Switches / Amplifiers / Routers

Backbone

Access / Metro Network

SA A D SD

SA A D SD

SA A D SD

SA A D SD

SA A D SD

SA A D SD

4. Which of the following PROTOCOLS topics should students learn?

Routing and Wavelength Assignment

Protection and Restoration

Medium Access

Switching (Packet, Burst)

Grooming

TCP/IP over WDM

MPLS

SA A D SD

SA A D SD

SA A D SD

SA A D SD

SA A D SD

SA A D SD

SA A D SD

5. Which of the following GENERAL PRINCIPLES topics should students learn? WDM, DWDM

SA A D SD

Layered / Hierarchical Network Architecture

SA A D SD

1st to 3rd Generation of Optical Systems

SA A D SD

6. What kind of materials / strategies would you use to support your teaching?

Textbook

Instructor Slides (Content)

Collection of Examples (Real Models, Animated Applets)

Workbook of Homework Problems

Solution Guide to Homework Problems

Database of Test Questions

List of Discussion Topics

SA A D SD

SA A D SD

SA A D SD

SA A D SD

SA A D SD

SA A D SD

SA A D SD

7. What would be the optimal delivery method for a graduate course in Optical networking?

Exclusively Face-to-Face Classroom Activities

SA A D SD

Emphasize Face-to-Face Classroom Activities with Online Readings and Assignments

SA A D SD

Half Online Activities and Half Classroom Activities

Emphasize Online Activities with Classroom Meetings

SA A D SD

SA A D SD

Entirely Online (Class Never Meets Face-to-Face)

SA A D SD

8. Overall, it is necessary to offer a graduate course in Optical networking to engineering students. SA A D SD 